\title{
First sequential images of the plasmasphere from the meridian perspective observed by KAGUYA
}

\author{
Go Murakami ${ }^{1}$, Ichiro Yoshikawa ${ }^{1}$, Yuki Obana ${ }^{1}$, Kazuo Yoshioka $^{1}$, Gentaro Ogawa $^{1}$, Atsushi Yamazaki ${ }^{2}$, \\ Masato Kagitani ${ }^{3}$, Makoto Taguchi ${ }^{4}$, Masayuki Kikuchi ${ }^{5}$, Shingo Kameda ${ }^{2}$, and Masato Nakamura ${ }^{2}$ \\ ${ }^{1}$ Department of Earth and Planetary Science, The University of Tokyo, 7-3-1 Hongo, Bunkyo, Tokyo 113-0033, Japan \\ ${ }^{2}$ Institute of Space and Astronautical Science, 3-1-1 Yoshinodai, Sagamihara, Kanagawa 229-8510, Japan \\ ${ }^{3}$ Planetary Plasma and Atmospheric Research Center, Tohoku University, Aramaki-aza-aoba, Aoba, Sendai 980-8578, Japan \\ ${ }^{4}$ Rikkyo University, 3-34-1 Nishi-Ikebukuro, Toshima, Tokyo 171-8501, Japan \\ ${ }^{5}$ National Institute of Polar Research, 10-3, Midoricho, Tachikawa, Tokyo 190-8518, Japan
}

(Received December 18, 2009; Revised January 24, 2010; Accepted February 20, 2010; Online published March 26, 2010)

\begin{abstract}
Our understanding of plasmaspheric dynamics has increased in recent years largely due to the information generated during the IMAGE-EUV mission. Even though this successful mission has ended, we have succeeded in imaging the terrestrial helium ions $\left(\mathrm{He}^{+}\right)$by the Telescope of Extreme Ultraviolet (TEX) aboard the Japanese lunar orbiter KAGUYA by detecting resonantly scattered emission at $30.4 \mathrm{~nm}$. The view afforded by the KAGUYA orbit encompasses the plasma $\left(\mathrm{He}^{+}\right)$distribution in a single exposure, enabling us to examine for the first time the globally averaged properties of the plasmasphere from the "side" (meridian) perspective. The TEX instrument observed a medium-scale density structure in the dawnside plasmasphere during a quiet period (1-2 June 2008). The meridian shape of the structure clearly agreed with the dipole magnetic field line. The TEX instrument also observed the structure in the plasmasphere co-rotating with a duration of $26 \mathrm{~h}$, which is consistent with results from a number of recent studies derived from the IMAGE-EUV mission. These results confirm that the TEX instrument successfully obtained the spatial distribution and temporal variation of the plasmasphere.

Key words: Plasmasphere, EUV, KAGUYA, corotation.
\end{abstract}

\section{Introduction}

The Earth's plasmasphere is a torus-shaped region filled with cold plasmas in the inner magnetosphere. Results obtained from conventional extreme ultraviolet (EUV) photometric experiments with an inside-out view, which were performed extensively in the 1970s, suggested that the Earth's plasmasphere would be imaged by He II $(30.4 \mathrm{~nm})$ emission (e.g., Johnson et al., 1971). In the 1990s, the remote sensing method using EUV emission became a powerful tool in the search for global perspectives on plasmasphere dynamics (Williams et al., 1992). The first plasmaspheric imaging was carried out by a small EUV scanner on board the Planet-B (Nozomi) spacecraft (Nakamura et al., 2000; Yoshikawa et al., 2000a, 2003) and proved that plasmaspheric EUV emission at $30.4 \mathrm{~nm}$ is bright enough to be taken in snapshots. The success of this first plasmaspheric EUV imaging provided our group with the incentive to construct global images of the geospace plasma distribution, namely, the plasmasphere, polar wind, and plasma sheet (Yoshikawa et al., 2000b, 2001a). A few years later, the Imager for magnetopause-to-Aurora Global Exploration (IMAGE) mission produced consecutive images of the terrestrial plasmasphere from the aerial view (Sandel et al., 2000; Burch et al., 2001). The IMAGE mission identified

Copy right(c) The Society of Geomagnetism and Earth, Planetary and Space Sciences (SGEPSS); The Seismological Society of Japan; The Volcanological Society of Japan; The Geodetic Society of Japan; The Japanese Society for Planetary Sciences; TERRAPUB.

doi:10.5047/eps.2010.02.006 not only conventional pictures of the plasmasphere (plasmapause, tails, and duskside bulge), but also novel structures, such as depleted regions that are called "voids" and a sharp azimuthal gradient in $\mathrm{He}^{+}$density called the "shoulder".

Our understanding of plasmaspheric dynamics is growing in many different aspects, such as how do convection electric fields penetrate into the body of plasmasphere? Murakami et al. (2007) investigated the plasmapause inward motion in response to the solar wind electric field, which was measured by solar wind monitor (ACE spacecraft). Their result is consistent with a lag time-scale derived from the ionospheric observations on the ground stations (e.g., Kikuchi et al., 1996; Ridley et al., 1998; Hashimoto et al., 2002). They concluded that the electric field penetrates from the magnetopause to the inner magnetosphere through the ionosphere.

Technological developments to visualize terrestrial EUV radiation at $30.4 \mathrm{~nm}$ are progressing (e.g., Yoshikawa et al., 2005), based on two rocket-borne experiments (Yoshikawa et al., 1997; Yamazaki et al., 2002), and we have built the second-generation extreme UV telescope for Japan's lunar mission (KAGUYA). The Upper atmosphere and Plasma Imager (UPI) on KAGUYA produces terrestrial images in the visible and EUV spectral ranges. Two telescopes are onboard; one is the Telescope for VISible emission (UPITVIS), and the other is our Telescope of EXtreme ultraviolet emission (UPI-TEX). UPI-TVIS takes pictures of the Earth's airglow and aurora at four wavelengths (Taguchi et al., 2009) and lunar sodium exosphere (Kagitani et al., 
2009). UPI-TEX images the resonance scattering emissions of oxygen ions (O II) at $83.4 \mathrm{~nm}$ and helium ion at 30.4 (He II) nm (Yoshikawa et al., 2008). KAGUYA was launched by the H-IIA rocket in 2007 and put into the orbit around the Moon, with the aim of producing EUV images from the meridian view (Obana et al., 2009). The KAGUYA project has carried out the scientific observations of the Moon, on the Moon, and from the Moon. In this paper, we report the initial results on terrestrial $\mathrm{He}$ II $(30.4 \mathrm{~nm})$ radiation obtained by the UPI-TEX instrument. This is the first observation of the inner magnetosphere (plasmasphere) from the meridian perspective. The novel images taken by the other EUV channel $(83.4 \mathrm{~nm})$ will be published as a separate paper.

\section{Instrumentation and Observation}

Imaging of the plasmasphere requires global 'snapshots' with wide field-of-view (FOV). KAGUYA provides researchers with an ideal platform that commands a panoramic view of geospace. The KAGUYA spacecraft was launched in September 2007 and entered into the lunar polar orbit at an altitude of $100 \mathrm{~km}$. The TEX instrument is mounted on two-rotational-axis gimbal system together with TVIS. The rotation around the azimuthal (AZ) axis cancels the apparent drift motion of the Earth in the image due to the orbital motion of the spacecraft. The other rotation (EL: elevation axis) cancels the orbital inclination of the Moon. Both rotations enable researchers to always keep the Earth in the center of the FOV. The TEX instrument is a type of normal-incidence telescope using a $\mathrm{Mo} / \mathrm{Si}$ multilayer coated mirror and a split thin metal filter, which was technologically identical to the past space-borne instruments (Yoshikawa et al., 1997, 2001b). The filter is made of aluminum/carbon and indium so as to be able to detect the resonance scattering emissions of He II and O II (Yoshikawa et al., 2008). The TEX instrument has a FOV of $8.4^{\circ} \times 8.4^{\circ}$, which corresponds to the $8.8 \mathrm{Re} \times 8.8 \mathrm{Re}$ at the Earth's position. The maximum spatial and temporal resolutions are $0.07 \mathrm{Re}$ and $1 \mathrm{~min}$, respectively.

The KAGUYA spacecraft orbits around the Moon with an orbital period of $2 \mathrm{~h}$. Therefore, TEX has an observation window every 2 , but the observation must satisfy geometrical conditions. Figure 1 shows the schematic geometry among the Earth, Moon, and the spacecraft. The instrument can be turned on under the following conditions: (1) KAGUYA is in view from the Earth; (2) KAGUYA is in the umbra of the Moon to reduce stray light to the UPI; (3) the umbra period is longer than 15 min because the UPI needs an intermission to rewind along the azimuthal axis every orbit. It is noted that TVIS observation needs one additional criterion, namely, (4) less than half of Earth's disk is sunshined.

After running primary function checks on the overall instrument, we had the opportunity to turn on the high voltage power supply on 1 May 2008. Unfortunately, the metal band-pass filter was mechanically damaged at this time, possible due to the vibration or shock at the launch. Two pinholes on the filter were identified. We investigated the exact locations of these pinholes during the EUV star calibration. A circular ghost appeared on all EUV images

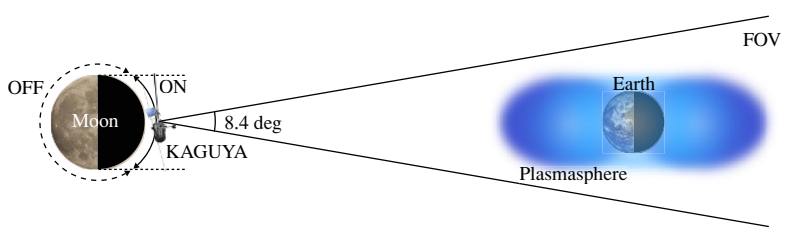

Fig. 1. Schematic diagram of the geometry among the Earth, Moon, and the KAGUYA spacecraft. TEX starts to observe under the conditions that (1) KAGUYA is in sight of the Earth, (2) KAGUYA is in the umbra of the Moon, and (3) the umbra period is longer than $15 \mathrm{~min}$.

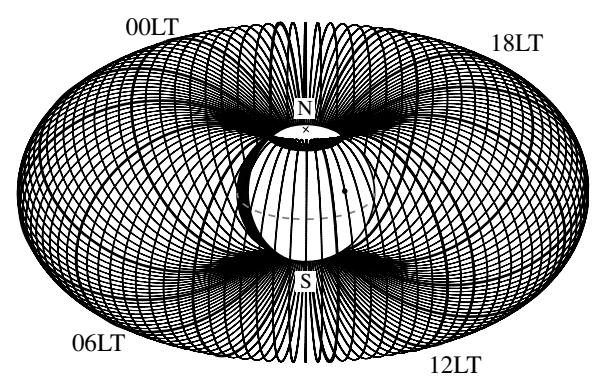

Fig. 2. View of the geomagnetic field lines seen from KAGUYA. During the observation in June 2008, our telescope gazed down to the Earth's geomagnetic equatorial plane at an angle of $20^{\circ}$. Each line indicates a dipolar magnetic field. The bold field lines are drawn so as to extend every 2 -h local time.

due to the leak of geocoronal emissions at the longer wavelength. The ghost location is stable in all EUV images and predictable by calculation, independent of plasmaspheric morphology, which simplifies the analysis and ensures that our study can be performed without additional care. In this paper, we have discarded all pixels that were potentially contaminated by the artificial structure.

Figure 2 shows a schematic drawing of geomagnetic field lines from the perspective view of KAGUYA. We should note that a two-dimensional view of the plasmasphere is not dipole-like, rather the outer boundary is elliptic because the brightness reflects the column density along the line-ofsight (LOS) of the measurement. During the observation in June 2008 (reported in the following section), our telescope gazed down onto the Earth's geomagnetic equatorial plane at an angle of $20^{\circ}$.

\section{Results and Discussion}

On 1-2 June 2008, the geomagnetic activity was quite low, and no geomagnetic storm had been identified since May 23. Figure 3 shows the geomagnetic conditions $\left(K_{\mathrm{p}}\right.$, $D_{\text {st }}$, and the AU and AL indices) and interplanetary magnetic field (IMF) $z$-component (in GSM coordinates) on 1-2 June 2008. The maximum $K_{\mathrm{p}}$ was 3 - during the observation and 3 during the previous $24-\mathrm{h}$ period. The $\mathrm{AU}$ and $\mathrm{AL}$ indices indicate that there were no significant substorms during this period. The IMF, measured by Advanced Composition Explorer (ACE) at the L1 libration point, clearly had a relatively northward tendency, with the exception of a very short period.

Figure 4 shows consecutive snapshots of the plasmasphere obtained by the TEX instrument on 1-2 June 2008. 


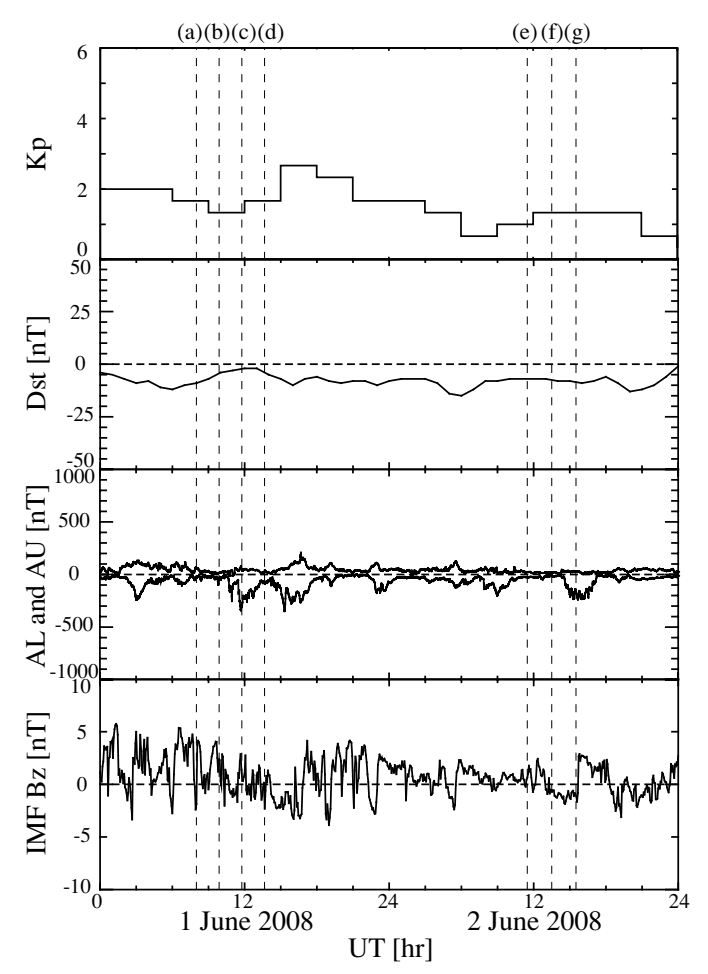

Fig. 3. Geomagnetic conditions $\left(K_{\mathrm{p}}, D_{\mathrm{st}}\right.$, and the $\mathrm{AU}$ and $\mathrm{AL}$ indices) and IMF $z$-component (in GSM coordinates) on 1-2 June 2008. In this period, the geomagnetic activity was quite low. Vertical dashed lines indicate our observation periods corresponding to each panel of Fig. 4. We used the IMF data measured by the MAG instrument onboard the ACE spacecraft. The IMF data are presented with a 44-min time delay.

To improve the signal-to-noise ratio of each image, (1) we accumulated all images obtained in the same revolution, and (2) we decreased the spatial resolution to $0.13 \mathrm{Re}$ using $2 \times$ 2 pixel binning. The Earth is located near the upper edge of each image. The dashed arc on the Earth represents the Earth's geomagnetic equator, and the dot on the Earth indicates the sub-solar point. During this period, the TEX instrument gazed down the Earth from the Moon, and the North Pole is presented as a cross mark in each image. The inner and outer contour lines are drawn at count rates of 2.0 and 1.0 count $/ \mathrm{min}$, respectively. The two white circles mask the circular ghosts explained in the previous section. For reference, the geomagnetic field lines $(L=3)$ are indicated with magnetic local time (MLT) in order to align the enhanced density structure. The magnetic field line in the consecutive images from Fig. 4(b-d) is identical, i.e., it co-rotates with the Earth (24-h duration). The magnetic field line described in Fig. 4(e-g) is also identical, but it is described with a 2-h lag period relative to that in Fig. 4(bd). The images of Fig. 4(b-d) were taken every 2-h, and the images of Fig. 4(e-g) were also taken with the same routine.

There seems to be no prominent feature in the midnightto-dawn region can be seen in Fig. 4(a) with the exception of the shadow. On the other hand, a medium-scale density structure, indicated by a black arrow, exists in the dawnside in Fig. 4(b-g). The shape of this structure inside the plasmasphere clearly agrees with the dipole magnetic field line. Although this structure could not be distinguished from our view direction as terms (e.g., finger and shoulder) discov-

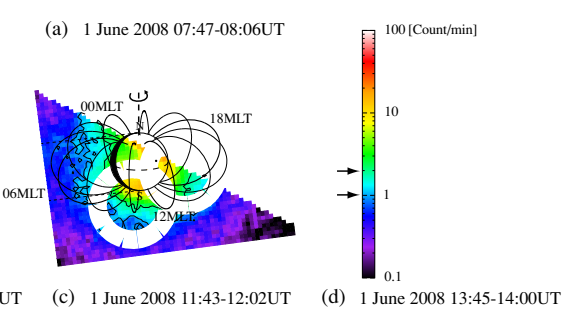

$\begin{array}{llll}\text { (b) } 1 \text { June } 2008 \text { 09:45-10:04UT } & \text { (c) 1 June 2008 11:43-12:02UT } & \text { (d) 1 June 2008 13:45-14:00UT }\end{array}$
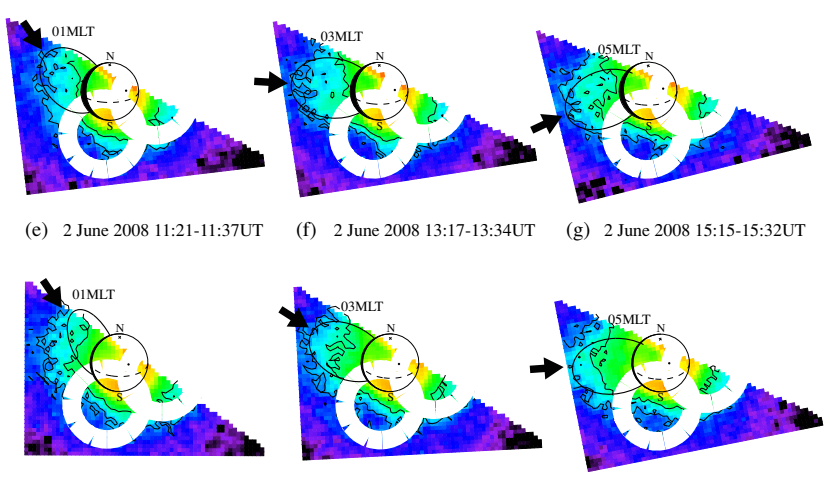

Fig. 4. Seven EUV images obtained by the TEX instrument on KAGUYA. The image was taken at 2-h intervals with the exception of the period between (d) and (e). The Earth is located near the upper edge of each image. The dashed arc on the Earth represents the Earth's geomagnetic equator, and the dot on the Earth is the sub-solar point. The inside and outside contour lines are drawn at count rates of 2.0 and 1.0 count $/ \mathrm{min}$, respectively. The two white circles are in place to mask the circular ghosts due to an instrument problem. The geomagnetic field lines $(L=3)$ are indicated at magnetic local time (MLT) to align the enhanced density structure. With the exception of image (a), an enhanced density structure, indicated by the black arrow, is clearly seen in each image. It is noted that the magnetic field line in the consecutive image from (b) to (d) is identical, i.e., it co-rotates with the Earth. The magnetic field line in the series of images from (e) to $(\mathrm{g})$ is also identical, but has a time lag of about $2 \mathrm{~h}$ from that in (b) to (d).

ered by the IMAGE spacecraft, this is the successful attempt at imaging a dipole-like density structure in the plasmasphere.

We are able to clearly see that the density structure rotated with the Earth in the dawnside. In Fig. 4(b), a part of the structure is likely to be in the Earth's night shadow; in Fig. 4(c) and (d), the structure has rotated endways together with the field line. While it passed through the dayside, it was difficult to be seen due to the high background luminosity (i.e., the main body of the plasmasphere) and its intersection with the ghost area (two white circles). No image is shown here for this period. The next day, the structure appeared in the dawnside and co-rotated with the Earth, as seen in Fig. 4(e-g). Comparisons of images Fig. 4(b-d) with Fig. 4(e-g) reveal that the duration is approximately $26 \mathrm{~h}$. (It should be remembered that the magnetic field line in the images from Fig. 4(e-g)) is not identical to that in the upper images, but has a lag period of 2-h.)

Sandel et al. (2003) found evidence that the main body of the plasmasphere does not always co-rotate with the Earth. They tracked notches, which are persistent distinctive lower density regions seen in EUV images obtained by the IMAGE spacecraft, and inferred the motion of particular volume elements of plasma. These researchers reported that the duration ranges between 26 and 31 hours. In a re- 
cent study, Galvan et al. (2008) found that the plasmasphere sub-corotates on average every $28 \mathrm{~h}$. Gallagher et al. (2005) studied the azimuthal motion of 18 notches seen in EUV pictures taken by the IMAGE spacecraft and derived a lag period in the range of $30 \mathrm{~min}$ to $4 \mathrm{~h}$. The duration in our EUV image seen from the meridian view is approximately $26 \mathrm{~h}$, which is not inconsistent with their conclusion based on the observation from the aerial view. This plasmasphere co-rotation lag is possibly caused by a corresponding co-rotation lag in the upper ionosphere, as suggested by Burch et al. (2004). These researchers compared rotation rates of plasmaspheric notches derived from EUV images to ionospheric drifts observed on DMSP spacecraft and found a good agreement between the two observations. They concluded that the cause of the co-rotation lag is the ionospheric disturbance dynamo.

\section{Conclusion}

EUV images from the meridian view have become available, even though the successful IMAGE-EUV mission has ended. These images will be mostly used to study plasmaspheric dynamics and products from IMAGE-EUV. The TEX instrument onboard the lunar orbiter (KAGUYA) clearly observed a dipole-like density enhancement in the dawnside plasmasphere during a quiet period. We have also presented the co-rotation of the near-Earth plasmas with the duration of $26 \mathrm{~h}$, which is consistent with results from recent studies derived from the IMAGE-EUV mission. We conclude that the TEX instrument successfully obtained the spatial distribution and temporal variation of the plasmasphere. In addition, we emphasize the necessity that a future convection model of the inner magnetosphere treats the phenomenon of plasmaspheric dynamics, as strongly stated by Burch et al. (2004).

Acknowledgments. The authors thank all members of the SELENE project team for their efforts in leading the successful mission.

\section{References}

Burch, J. L., S. B. Mende, D. G. Mitchell, T. E. Moore, C. J. Pollock, B. W. Reinisch, B. R. Sandel, S. A. Fuselier, D. L. Gallagher, J. L. Green, J. D. Perez, and P. H. Reiff, Views of Earth's magnetosphere with the IMAGE satellite, Science, 291, 619-624, 2001.

Burch, J. L., J. Goldstein, and B. R. Sandel, Cause of plasmasphere corotation lag, Geophys. Res. Lett., 31, doi:10.1029/2003GL019164, 2004.

Gallagher, D. L., M. L. Adrian, and M. W. Liemohn, Origin and evolution of deep plasmaspheric notches, J. Geophys. Res., 110(A9), doi:10. 1029/2004JA010906, 2005.

Galvan, D. A., M. B. Moldwin, and B. R. Sandel, Diurnal variation in plasmaspheric $\mathrm{He}^{+}$inferred from extreme ultraviolet images, J. Geophys. Res., 113(A9), doi:10.1029/2007JA013013, 2008.

Hashimoto, K. K., T. Kikuchi, and Y. Ebihara, Response of the magnetospheric convection to sudden interplanetary magnetic field changes as deduced from the evolution of partial ring currents, J. Geophys. Res., 107(A11), 1337, doi:10.1029/2001JA009228, 2002.

Johnson, C. Y., J. M. Young, and J. C. Holmes, Magnetoglow-A new geophysical resource, Science, 171, 379, 1971.

Kagitani, M., M. Taguchi, A. Yamazaki, I. Yoshikawa, G. Murakami, K. Yoshioka, S. Kameda, F. Ezawa, T. Toyota, and S. Okano, First optical observation of the Moon's sodium exosphere from the lunar orbiter SELENE (Kaguya), Earth Planets Space, 61, 1025, 2009.

Kikuchi, T., H. Lühr, T. Kitamura, O. Saka, and K. Schlegel, Direct penetration of the polar electric field to the equator during a DP2 event as detected by the auroral and equatorial magnetometer chains and the EISCAT radar, J. Geophys. Res., 101, 17161, 1996.

Murakami, G., M. Hirai, and I. Yoshikawa, The plasmapause response to the southward turning of the IMF derived from sequential EUV images, J. Geophys. Res., 112, A06217, doi:10.1029/2006JA012174, 2007.

Nakamura, M., I. Yoshikawa, A. Yamazaki, K. Shiomi, Y. Takizawa, M. Hirahara, K. Yamashita, Y. Saito, and W. Miyake, Terrestrial plasmaspheric imaging by an Extreme Ultraviolet Scanner on planet-B, Geophys. Res. Lett., 27(2), 141, 2000.

Obana, Y., G. Murakami, I. Yoshikawa, I. R. Mann, P. J. Chi, and M. B. Moldwin, Conjunction study of plasmapause location using groundbased magnetometers, IMAGE-EUV, and KAGUYA-TEX data, J. Geophys. Res., 2009 (in press).

Ridley, A. J., G. Lu, C. R. Clauer, and V. O. Papitashvili, A statistical study of the ionospheric convection response to changing interplanetary magnetic field conditions using the assimilative mapping of ionospheric electrodynamics technique, J. Geophys. Res., 103(A3), 4023, 1998.

Sandel, B. R., A. L. Broadfoot, C. C. Curtis, R. A. King, T. C. Stone, R. H. Hill, J. Chen, O. H. W. Siegmund, R. Raffanti, D. D. Allred, R. S. Turley, and D. L. Gallagher, The Extreme Ultraviolet Imager investigation for the IMAGE mission, Space Sci. Rev., 91(1/2), 197-242, 2000.

Sandel, B. R., J. Goldstein, D. L. Gallagher, and M. Spasojevic, Extreme Ultraviolet Imager observations of the structure and dynamics of the plasmasphere, Space Sci. Rev., 109, 25, 2003.

Taguchi, M., T. Sakanoi, S. Okano, M. Kagitani, M. Kikuchi, M. Ejiri, I Yoshikawa, A. Yamazaki, G. Murakami, K. Yoshioka, S. Kameda, W. Miyake, M. Nakamura, and K. Shiokawa, The Upper Atmosphere and Plasma Imager/the Telescope of Visible Light (UPI/TVIS) onboard the Kaguya spacecraft, Earth Planets Space, 61, xvii, 2009.

Williams, D. J., E. C. Roelof, and D. G. Mitchell, Global magnetospheric imaging, Rev. Geophys., 30, 183, 1992.

Yamazaki, A., S. Tashiro, Y. Nakasaka, I. Yoshikawa, W. Miyake, and M. Nakamura, Sounding-rocket observation of O II 83.4-nm emission over the polar ionosphere, Geophys. Res. Lett., 29(21), doi:10. 1029/2002GL014788, 2002.

Yoshikawa, I., M. Nakamura, M. Hirahara, Y. Takizawa, K. Yamashita, H. Kunieda, T. Yamazaki, K. Misaki, and A. Yamaguchi, Observation of He II emission from the plasmasphere by a newly developed EUV telescope on board sounding rocket S-520-19, J. Geophys. Res., 102, 19897-19902, 1997.

Yoshikawa, I., A. Yamazaki, K. Shiomi, K. Yamashita, Y. Takizawa, and M. Nakamura, Evolution of the outer plasmasphere during low geomagnetic activity observed by the EUV scanner onboard Planet-B, J. Geophys. Res., 105, 27777-27789, 2000a.

Yoshikawa, I., A. Yamazaki, K. Shiomi, K. Yamashita, Y. Takizawa, and M. Nakamura, Photometric measurement of cold helium ions in the magnetotail by an EUV scanner onboard Planet-B: Evidence of the existence of cold plasmas in the near-Earth plasma sheet, Geophys. Res. Lett., 27(21), 3567-3570, 2000b.

Yoshikawa, I., A. Yamazaki, K. Shiomi, M. Nakamura, K. Yamashita, Y. Saito, M. Hirahara, Y. Takizawa, W. Miyake, and S. Matsuura, Development of a compact EUV photometer for imaging the planetary magnetosphere, J. Geophys. Res., 106(A-11), 26057-26074, 2001a.

Yoshikawa, I., A. Yamazaki, K. Shiomi, K. Yamashita, Y. Takizawa, and M. Nakamura, Interpretation of the He II (304A) EUV image of the inner magnetosphere by using empirical models, J. Geophys. Res., 106, 25745-25758, 2001b.

Yoshikawa, I., A. Yamazaki, K. Yamashita, Y. Takizawa, and M. Nakamura, Which is a significant contributor for outside of the plasmapause, an ionospheric filling or a leakage of plasmaspheric materials?:

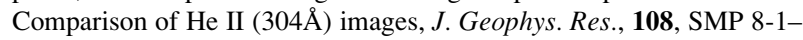
8-8, 2003.

Yoshikawa, I., T. Murachi, H. Takenaka, and S. Ichimaru, Multilayer coating for $30.4 \mathrm{~nm}$, Rev. Sci. Instrum., 76(6), 066109-1-2, 2005.

Yoshikawa, I., A. Yamazaki, G. Murakami, K. Yoshioka, S. Kameda, F. Ezawa, T. Toyota, W. Miyake, M. Taguchi, M. Kikuchi, and M. Nakamura, Telescope of extreme ultraviolet (TEX) onboard SELENE: science from the Moon, Earth Planets Space, 60, 407-416, 2008.

G. Murakami (e-mail: go@eps.s.u-tokyo.ac.jp), I. Yoshikawa, Y. Obana, K. Yoshioka, G. Ogawa, A. Yamazaki, M. Kagitani, M. Taguchi, M. Kikuchi, S. Kameda, and M. Nakamura 